Conclusions A comprehensive integrated analysis of safety outcomes in a large, well-defined cohort of 1299 patients with IPF who were treated with pirfenidone for up to 9.9 years demonstrated that treatment with pirfenidone is safe and generally well tolerated. These observations provide further evidence to support the long-term clinical safety of pirfenidone in patients with IPF.

\section{P14 PIRFENIDONE IS EFFICACIOUS IN PATIENTS WITH IDIOPATHIC PULMONARY FIBROSIS (IPF) WITH MORE PRESERVED LUNG FUNCTION}

${ }^{1} \mathrm{PW}$ Noble, ${ }^{2} \mathrm{WZ}$ Bradford, ${ }^{3} \mathrm{U}$ Costabel, ${ }^{4}$ G Glaspole, ${ }^{5} \mathrm{MK}$ Glassberg, ${ }^{2} \mathrm{E}$ Gorina, ${ }^{2} \mathrm{D}$ Kardatzke, ${ }^{6} \mathrm{~L}$ Lancaster, ${ }^{7} \mathrm{DJ}$ Lederer, ${ }^{8} \mathrm{SD}$ Nathan, ${ }^{9} \mathrm{C}$ Pereira, ${ }^{10} \mathrm{D}$ Spirig, ${ }^{11} \mathrm{JJ}$ Swigris, ${ }^{12} \mathrm{D}$ Valeyre, ${ }^{13} \mathrm{C}$ Albera. ${ }^{1} \mathrm{C}$ dedars-Sinai Medical Center, Los Angeles, California, USA; IInterMune, Inc. (A Wholly Owned Roche Subsidiary), Brisbane, California, USA; ${ }^{3}$ Ruhrlandklinik and University of Duisburg - Essen, Essen, Germany; ${ }^{4}$ Alfred Hospital, Melbourne, Australia; ${ }^{5}$ University of Miami Miller School of Medicine, Miami, Florida, USA; ${ }^{6}$ Vanderbilt University Medical Center, Nashville, Tennessee, USA; ${ }^{7}$ Columbia University Medical Center, New York, New York, USA; ${ }^{8}$ Inova Fairfax Hospital, Falls Church, Virginia, USA; ${ }^{9}$ Paulista School of Medicine, Federal University of São Paulo, São Paulo, Brazil; ${ }^{10} \mathrm{~F}$. Hoffman-La Roche Ltd, Basel, Switzerland; " National Jewish Health, Denver, Colorado, USA; ${ }^{12}$ Assistance Publique-Hôpitaux de Paris, Hôpital Avicenne, Bobigny, France; ${ }^{13}$ University of Turin, Turin, Italy

\subsection{6/thoraxjnl-2015-207770.151}

Introduction and objectives IPF is a progressive, irreversible and fatal disease. Early treatment initiation when lung function is relatively preserved may have beneficial outcomes; however, published data to support this hypothesis are lacking. We investigated the efficacy of pirfenidone at 12 months in patients stratified by lung function using forced vital capacity (FVC) or GAP stage.

Methods Efficacy outcomes (FVC, 6-minute walk distance [6MWD] and dyspnea [UCSD SOBQ]) were analysed at 12 months in patients randomised to pirfenidone $2403 \mathrm{mg} / \mathrm{d}$ or placebo in the pooled CAPACITY/ASCEND population $(\mathrm{N}=$ 1247), stratified by baseline FVC $(\geq 80 \%,<80 \%)$ and GAP stage (GAP I, GAP II-III). Treatment-by-subgroup interaction was tested based on a rank ANCOVA model. The factors in the model included study, region, treatment, subgroup and treatment-by-subgroup interaction term.

Results Demographic characteristics were similar across all four groups. In the placebo arm, disease progression as measured by FVC occurred with comparable frequency in patients with FVC $\geq 80 \%$ and FVC $<80 \%$, as well as in patients with GAP I and GAP II-III stage. A higher proportion of placebo patients with FVC $<80 \%$ and GAP II-III stage had a $\geq 50-m$ decline in $6 \mathrm{MWD}$ or death or a $\geq 20$-point change in the UCSD SOBQ total score. Pirfenidone treatment reduced the proportion of patients experiencing a $\geq 10 \%$ FVC decline or death and increased the proportion of patients with no FVC decline in all subgroups. Pirfenidone also reduced the proportion of patients with $\geq 50-\mathrm{m}$ decline in the $6 \mathrm{MWD}$ or death and increased the proportion of patients with no 6MWD decline in all subgroups. The magnitude of treatment effect in patients with less $v s$ more preserved lung function was comparable, with no significant treatment-by subgroup interaction (Figure 1).

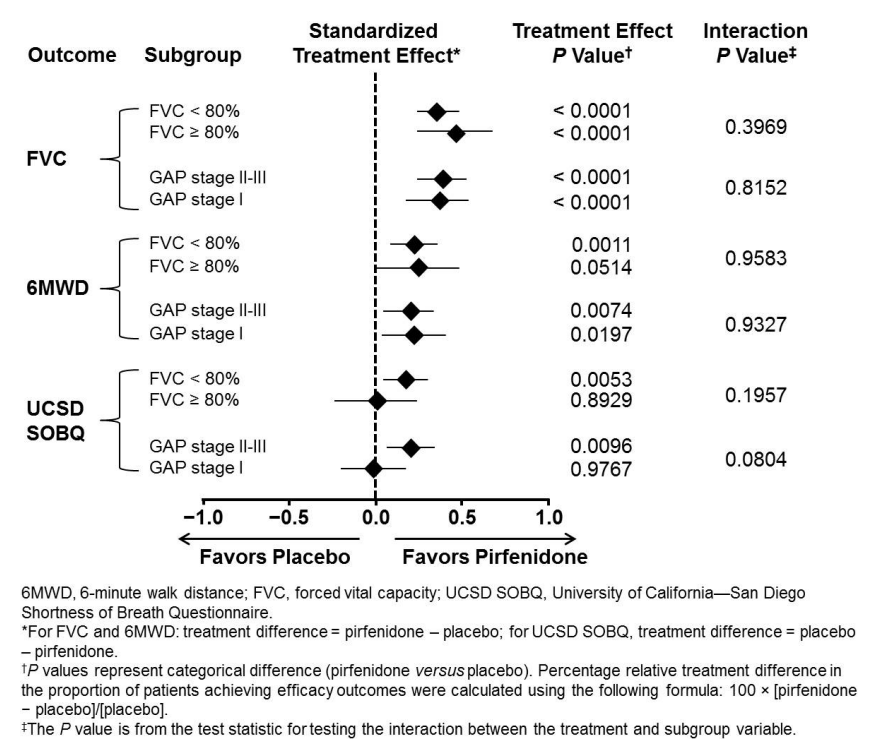

Abstract P14 Figure 1 Treatment effect of pirfenidone by baseline lung function

Conclusions In the placebo population, clinically significant disease progression occurs in subgroups with more and less preserved lung function at baseline, underlying the need for early intervention. The magnitude of pirfenidone treatment effect on functional measures was comparable in these subgroups of patients (FVC $<80 \%$ vs FVC $\geq 80 \%$ or GAP I vs GAP II-III stage), supporting the initiation of treatment soon after diagnosis, when pulmonary function is relatively preserved.

\section{Clinical studies of advanced COPD}

\section{P15 REGIONAL CEREBRAL ATROPHY AND COGNITIVE FUNCTION IN CHRONIC OBSTRUCTIVE PULMONARY DISEASE}

${ }^{1} \mathrm{CC}$ Savage, ${ }^{1} \mathrm{C}$ Pennington, ${ }^{2} \mathrm{PW}$ Jones, ${ }^{1} \mathrm{JW}$ Dodd. ${ }^{1}$ University of Bristol, Bristol, UK; ${ }^{2} \mathrm{St}$ George's University of London, London, UK

\subsection{6/thoraxjnl-2015-207770.152}

Background Widespread white matter damage and cognitive impairment have been demonstrated in COPD. However, it remains unclear if regional atrophy is present. We used a simple clinical visual rating scale to measure regional atrophy in a wellcharacterised population with COPD and compared age-matched controls. We explored correlations with demographics, disease factors and cognitive measures.

Objectives

1. a) To determine if there are significant differences in regional atrophy between COPD patients and age-matched control subjects.

2. b) To investigate whether patient characteristics or measures of disease severity account for group differences in atrophy severity.

3. c) To seek correlations with regional atrophy.

Methods A validated visual analogue MRI grading technique was used to assess the degree of atrophy in multiple brain regions in stable non-hypoxaemic COPD patients $(\mathrm{n}=25)$ and age- 